\title{
Bilateral adrenal lymphoma with adrenal insufficiency: a case report with litretures review
}

\begin{abstract}
Lymphoma arising in and confined to the adrenal glands is unusual and is termed primary adrenal lymphoma. Bilateral primary adrenal nonHodgkin's lymphoma is rare. We report 58-year-old woman with bilateral primary adrenal lymphoma and adrenal insufficiency.
\end{abstract}

Keywords: bilateral primary adrenal lymphoma, adrenal insufficiency

\author{
Volume 6 Issue 2 - 2018 \\ Muneera A Al Shareef,' Khalid S Al Jabri,' \\ Saeed J Alzahrani, ${ }^{2}$ Samia A Bokhari,' \\ Mohamed Abaza, ${ }^{3}$ Saeed A Bajabaa ${ }^{4}$ \\ 'Departments of Endocrinology, King Fahd Armed Forces \\ Hospital, Saudi Arabia \\ 2Department of Radiology, King Fahd Armed Forces Hospital, \\ Saudi Arabia \\ ${ }^{3}$ Department of Pathology, King Fahd Armed Forces Hospital, \\ Saudi Arabia \\ ${ }^{4}$ Department of Internal Medicine, King Fahd Armed Forces \\ Hospital, Saudi Arabia
}

\begin{abstract}
Correspondence: Khalid S Aljabri, FACP,ABIM,ABEM, Department of Endocrinology, King Fahad Armed Forces Hospital, PO Box 9862, Jeddah 21 I59, Jeddah, Kingdom of Saudi Arabia, Tel +966590008035, Fax +96625760665, Email khalidsaljabri@yahoo.com
\end{abstract}

Received: February 16, 2018 | Published: April 17, 2018

\section{Introduction}

Adrenal masses usually metastase from malignant tumours. ${ }^{1}$ NonHodgkin's lymphoma affecting the adrenal glands is usually associated with other sites of disease. ${ }^{2}$ Adrenal involvement in widespread nonHodgkin's lymphoma occured in $4 \%$ of cases as assessed by computed tomography and in $24 \%$ at postmortem examination. ${ }^{3,4}$ Disease arising in and confined to the adrenal glands is unusual and termed primary adrenal lymphoma. Bilateral primary adrenal nonHodgkin's lymphoma is rare with less than 200 reported cases..$^{5-12}$ We reported a 58 years old woman with bilateral adrenal lymphoma and adrenal insufficiency.

\section{Case report}

58-year-old woman referred to our endocrine clinic with bilateral adrenal mass found incidentally on computed tomography (CT) of the abdomen during a workup for abdominal pain. She has a history of abdominal pain associated with decreased appetite, marked weight loss and fever with night sweating for 1 year which had been progressive over the last few months,. No history of paroxysmal attacks of palpitation or headache, nor history of hirsutism. Past medical history was rlevant for type 2 diabetes mellitus; hypertension and she had hypoglycemic attacks, postural dizziness. She was off her antihypertensive medications and oral hypoglycemic medications for 1-month prior to presentation. On clinical examination, she was pale. Blood pressue $124 / 60$, and pulse $85 /$ minute and regular. No skin pigmentation, no lymph node enlargement. Her abdomen was soft, with no tenderness or organomegaly. Laboratory investigations showed a hemoglobin of $8.1 \mathrm{~g} / \mathrm{dL}$, Hemoglobin; A1c 4.9, sodium and potassium were normal, Erythrocyte sedimentation rate; 120 $\mathrm{mm} / \mathrm{hr}(<20 \mathrm{~mm} / \mathrm{hr})$, Lactic acid dehydrogenase; $1400 \mathrm{IU} / \mathrm{L}$ (250 and $500 \mathrm{IU} / \mathrm{l})$. CT abdomen showed large, well defined with homogeneous enhancement bilateral adrenal masses $7.5 \times 4.9 \mathrm{~cm}$ and nor hemorrhage or calcification were seen with large para-aortic lymph node $4.4 \mathrm{~cm}$ (Figure 1). The adrenal masse work up showed: Aldosterone $3.7 \mathrm{ng} / \mathrm{dL}$ (3.5-37.1), Renin $4.2 \mathrm{ng} / \mathrm{ml} / \mathrm{L}$ (0.76-4.61), Plasma free metanephrine $\leq 0.03 \mathrm{nmol} / \mathrm{L}(0.03-0.85)$, Pasma free normetanephrine $0.44 \mathrm{nmol} / \mathrm{L}(0.04-1.39)$, Dehydroepiandrosulf (DHEA-S $\leq 0.8 \mathrm{mmol} / \mathrm{L} \quad(0.8-4.9)$. Patient clinical condition deteriorated and admitted with severe abdominal pain, vomiting and hypotension refractory to the intravenous fluid. Adrenal insufficiency was highly suspected. Random serum cortisol was extracted and hydrocortisone I.V was started. Random serum cortisol level was 59 nmol/L (171-536). No Adrenocorticotropic hormone (ACTH) level was obtained. Short synacthen test was not done because of rapid deterioration of her clinical condition. Chest X-ray showed bilateral pleural effusion, aspiration cytology of the pleural fluid showed atypical large lymphocytes, suspicious of malignant lymphoma. Fine needle aspiration from Left para-aortic mass was consistent with diffuse large B-cell lymphoma (Figure 2A) (Figure 2B). Patient condition deteriorated further, and she passed away.

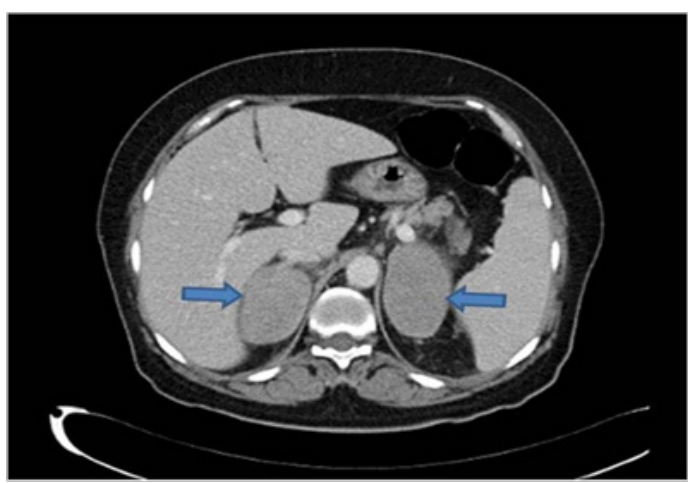

Figure I CT scan of abdomen (Portal venous phase axial) showed large, well defined with homogeneous enhancement bilateral large adrenal masses (blue arrows), nor hemorrhage or calcification were shown. 


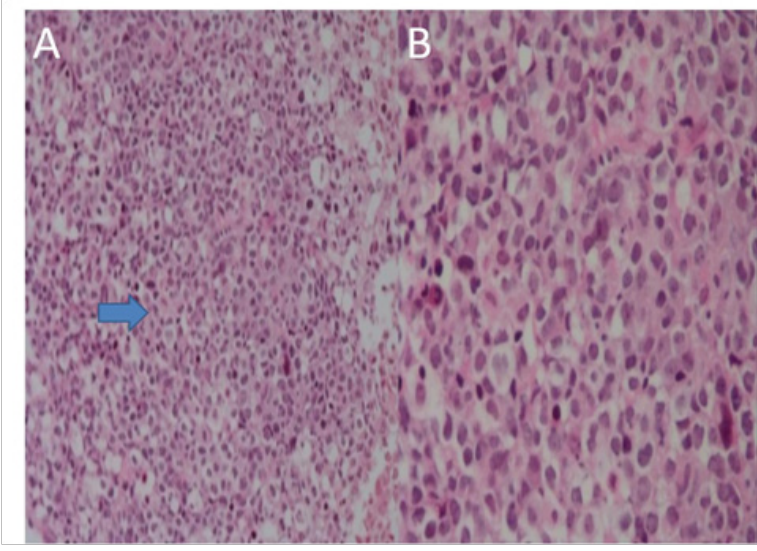

Figure 2 Diffuse large atypical lymphoid cells. A: $\mathrm{H}$ and E; $200 X$ (blue arrow), B: $400 X$.

\section{Discussion}

Adrenal masses are common and regularly detected as incidental lesions. The prevalence of adrenal incidentaloma varies among the literature. However, all become rather common with the use of radiographic images in clinical practice. ${ }^{13}$ The differential diagnosis of bilateral adrenal lesions includes adrenal metastasis, lymphoma, bilateral pheochromocytoma, adrenocortical carcinoma, macronodular adrenal hyperplasia; granulomatous disease and adrenal hemorrhage. Bilateral distribution and other imaging traits: wash-out estimation, the presence of calcification, lesion magnitude, including clinical and hormonal assessment can assist narrowing the differential diagnoses in case of adrenal masses. ${ }^{13,14}$ Adrenal gland neoplasm can be a metastasis from other organs that is greater common than primary adrenal neoplasm. ${ }^{15}$ Lymphoma arises in particular rare extranodal organs with or without adjacent lymph node swelling considered to be a reasonable definition for primary extranodal lymphoma as described in some literature which can be applied for primary adrenal lymphoma as well as our case showed. ${ }^{16,17}$

Primary adrenal lymphoma (PAL) is very rare tumors with less than 200 reported cases, when as non-Hodgkin's lymphoma; PAL reported to be less than $1 \%$ with $70 \%$ of cases were bilateral. ${ }^{18}$ Immune disorders, Epstein-Barr virus infection, and mutations in the p53 and c-kit genes are thought to make contributions in the pathogenesis of PAL. ${ }^{19}$ Background of preceding autoimmune adrenalitis and direct infiltration by neoplasm are mechanisms which could explain the adrenal insufficiency that is regularly observed in patients with PAL, The non-particular symptoms and signs of adrenal insufficiency may mask its early diagnosis, and patients may present with unrecognized adrenal crisis. $^{20}$

PAL adrenal insufficiency is likely to happen if more than $90 \%$ adrenal tissue involved by the neoplasm and occurrence of Addisonian emergency can prompt to extreme life-threatening outcomes, therefore substitution therapy need to be taken into consideration of PAL. ${ }^{21}$ The case in the current report had extensive bilateral infiltration the adrenal gland by the tumor. Adrenal insufficiency in our case diagnosed by the refractory hypotension, very low cortisol although short synacthen test was not done because the patient's condition, a cutoff threshold for basal cortisol concentrations of $140 \mathrm{nmol} / \mathrm{L}(\leq 5 \mu \mathrm{g} / \mathrm{dL})$ drawn in the morning (6 to $10 \mathrm{AM}$ ) is suggestive of adrenal insufficiency, also low serum DHEA-S are very useful when adrenal insufficiency is suspected. ${ }^{22,23}$

The commonest histological type of lymphoma seen in PAL is High-grade diffuse large B-cell lymphoma ( $78 \%$ of cases). These types of tumors are considered to be fatal, poor prognostic criteria are old age, large tumor, high level of lactate dehydrogenase and adrenal insufficiency on presentation..$^{24,25}$ The response to the therapeutic modalities depend on the presentation, the multiple options of treatment. Management options for PAL include surgery, radiation, and chemotherapy. Currently, chemotherapy is the mainstay in the management of PAL, principally the traditional regimens (CHOP), with the inclusion of different modalities as rituximab, and laparoscopic surgery as an adjuvant to chemotherapy for masses larger size masses. In spite of that medical responses have been accomplished primarily in patients with early stages, prognosis is poor and average survival is 12.5 weeks to 6 months. Hatjiharissi et al. the patient described had most of the poor prognostic factors and deteriorated rapidly and passed away before starting chemotherapy regimen.

\section{Conclusion}

PAL is to a great degree uncommon tumor. Early diagnosis of adrenal insufficiency is essential to start the appropriate therapy.

\section{Acknowledgment}

None.

\section{Conflict of interest}

None.

\section{References}

1. Glomset DA. The incidence of metastasis of malignant tumours to the adrenals. Am J Cancer. 1938;32:57-61.

2. Glazer HS, Lee JK, Balfe DM. Non-Hodgkin's lymphoma: computed tomography demonstration of unusual extranodal involvement. Radiology. 1983;149(1):211-217.

3. Paling MR, Williamson BRJ. Adrenal involvement in non-Hodgkin's lymphoma. AJR Am J Roentgenol. 1983;141(2):303-305.

4. Rosenberg SA, Diamond HD, Jaslowitz B, et al. Lymphosarcoma: a review of 1269 cases. Medicine (Baltimore). 1961;40:31-84.

5. Feldberg MA, Hendriks MJ, Klinkhammer AC. Massive bilateral nonHodgkin's lymphoma of the adrenals. Urol Radiol. 1986;8(2):85-88.

6. Ito Y, Yamaha M, Koide T, et al. A case of bilateral adrenal non- Hodgkin's lymphoma. Acta Urol Jpn. 1988;34:137-140.

7. Aron E, Jobard P, Groussin P, et al. Bilateral and primary lymphosarcoma of the adrenal glands. Semaine des Hopitaux. 1971;47(53):3067-371.

8. Pagliuca A, Gillett DS, Salisbury JR, et al. Bilateral adrenal lymphoma presenting as Addison's disease. Postgrad Med J. 1989;65(767):684-686.

9. Domenici A. Bilateral destructive lymphosarcoma of the adrenals. Pathologica. 1948;40:104

10. Shea TC, Spark R, Kane B, et al. Non-Hodgkin's lymphoma limited to the adrenal gland with adrenal insufficiency. Am J Med 1985;78(4):711-714.

11. Sparagana M. Addison's disease due to reticulum cell sarcoma apparently limited to the adrenals. J Am Geriatric Soc. 1970;18(7):550-554. 
12. Alvarez-Costello A. Computed tomography of primary lymphoma of the adrenal area. J Ultrasound Med. 1993;17:408-409.

13. Glazer HS, Weyman PJ, Sagal SS, et al. Nonfunctioning Adrenal masses: incidental discovery on computed tomography. Am J Roentgenol. 1982;139(1):81-85.

14. Dhamija E, Panda A, Das CJ, et al. Adrenal imaging (Part 2): Medullary and secondary adrenal lesions. Indian J Endocrinol Metab. 2015;19(1):16-24.

15. Wu H, Shih L, Chen T. A patient with bilateral primary adrenal lymphoma, presenting with fever of unknown origin and achieving long-term diseasefree survival after resection and chemotherapy. Ann Hematol. 1999; 78(6):289-292.

16. Ostrowski ML, Unni KK, Banks PM, et al. Malignant lymphoma of bone. Cancer. 1986;58(12):2646-2655.

17. Wiseman C, Liao KT. Primary lymphoma of the breast. Cancer. 1972;29(6):1705-1712.

18. Rashidi A, Fisher SI. Primary adrenal lymphoma: a systematic review. Ann Hematol. 2013;92(12):1583-1593.

19. Padhi S, Sahoo J. Primary adrenal non Hodgkin lymphoma: changing trends. Turk J Gastroenterol. 2015;26(1):85-86.
20. Simpson WG, Babbar P, Payne LF. Bilateral primary adrenal nonHodgkin's lymphoma without adrenal insufficiency. Urol Ann. 2015;7(2):259-261.

21. De Miguel Sánchez C, Ruiz L, González JL, et al. Acute adrenal insufficiency secondary to bilateral adrenal B-cell lymphoma: a case report and review of the literature. Ecancermedicalscience. 2016;10:634.

22. Kazlauskaite R, Evans AT, Villabona CV, et al. Corticotropin tests for hypothalamic-pituitary-adrenal insufficiency: a metaanalysis. $J$ Clin Endocrinol Metab. 2008;93(11):4245-4253.

23. Al-Aridi R, Abdelmannan D, Arafah BM. Biochemical diagnosis of adrenal insufficiency: the added value of dehydroepiandrosterone sulfate measurements. Endocr Pract. 2011;17(2):261-270.

24. Libe R, Giavoli C, Barbetta L, et al. A primary adrenal non-Hodgkin's lymphoma presenting as an incidental adrenal mass. Exp Clin Endocrinol Diabetes. 2006;114(3):140-144.

25. Kim Y, Kim J, Min Y, et al. Prognostic factors in primary diffuse large B-cell lymphoma of adrenal gland treated with rituximab-CHOP chemotherapy from the Consortium for Improving Survival of Lymphoma (CISL). J Hematol Oncol. 2012;5:49. 